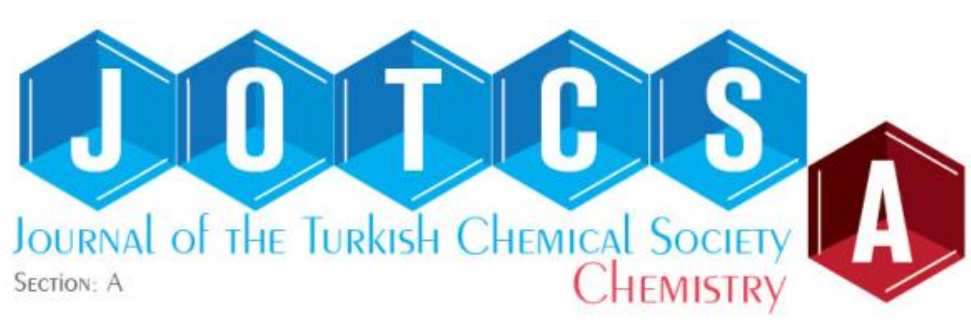

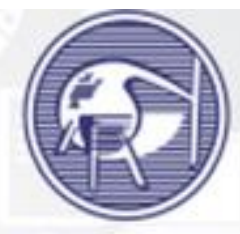

TURKISH

CHEMICAL SOCIETY

\title{
Optical investigation of palladium(II) phthalocyanine including an aromatic group
}

\author{
İbrahim Özçeşmeci ${ }^{* *}$ and İdris Sorar ${ }^{\text {b* }}$ \\ a Istanbul Technical University, Department of Chemistry, Maslak, TR-34469, Istanbul, Turkey \\ b Mustafa Kemal University, Department of Physics, Antakya, TR-31040, Hatay, Turkey
}

\begin{abstract}
Peripherally $\beta$-naphthoxy-substituted palladium(II) phthalocyanine was synthesized from corresponding phthalonitrile compounds. The palladium(II) phthalocyanine was characterized with ultraviolet-visible spectroscopy (UV-Vis), fourier transform-infrared spectroscopy (FT-IR), mass spectrometry, and elemental analysis techniques. Thin films of palladium(II) phthalocyanine were prepared using different organic solvents by spin coating technique. Transmittance and absorbance spectra of the thin films were studied in the wavelength range of $200-1000 \mathrm{~nm}$. Optical band gaps of palladium(II) phthalocyanine thin films were also calculated.
\end{abstract}

Keywords: Phthalocyanine, $\beta$-naphthol, palladium, thin film, optical properties.

Submitted: October 03, 2017. Accepted: January 12, 2018.

Cite this: Özçeşmeci İ, Sorar İ. Optical investigation of palladium(II) phthalocyanine including an aromatic group. JOTCSA. 2018;5(1):295-302.

DOI: http://dx.doi.org/10.18596/jotcsa.341381.

Corresponding Author Fax: +90 (212) 2856386; Tel.: +90 (212) 2857309

e-mail : ozcesmecii@itu.edu.tr

Corresponding Author Fax: +90 (326) 2455867; Tel.: +90 (326) 2455845

e-mail : sorar@mku.edu.tr 


\section{INTRODUCTION}

Phthalocyanines (Pcs) are macrocyclic compounds which are highly stable against elevated temperatures and light. The colors of Pcs range from dark blue to dark green. They attracted great interest due to their use as pigments. The electronic, optical, structural, and coordination properties of Pcs and their ability to be modified according to purpose have created quite different application areas for them than their classical uses. These applications include information technology, semiconductors, photodynamic agents, electrochromic devices, liquid crystalline materials, molecular materials and nonlinear optical materials, Langmuir-Blodgett films, and many catalytic processes $(1,2)$. Most of these applications are related to the planar $\Pi$ configuration system of Pc as much as the structure of the central metal atom. The addition of substituents to the peripheral positions increases the distance between the Pc conjugated $18-\Pi$ electron systems and facilitates their solubility. This allows the optical and optoelectronic properties of the molecule to be adjusted $(3,4)$.

There are many acquisition methods for thin films used in different technological fields. The coating techniques are divided into four basic methods in consideration of the physical condition of the coating material and the surface of the coating. These methods are coatings made from solid state, made from solution, made from liquid or semi-liquid state, and made from vapor phase (5). The spin coating method is one of the most commonly used processes for obtaining thin films of soluble Pc compounds containing substituent groups $(6,7)$. In this method, the final film thickness and other properties of thin film depend on the solution properties (concentration, drying speed, solid ratio, and surface tension) and processing conditions (rotation speed) $(8,9)$.

In this work, we have synthesized and characterized peripherally $\beta$-naphthoxy unit substituted palladium(II) phthalocyanine. We have also obtained thin films of palladium(II) phthalocyanine using different organic solvents [chloroform (CF), tetrahydrofuran (THF), dimethylformamide (DMF) and dimethyl sulfoxide (DMSO)] with spin coating technique and investigated their optical properties. Besides, optical band gaps of palladium(II) phthalocyanine thin films were also calculated.

\section{EXPERIMENTAL}

\section{Equipments and Materials}

FT-IR spectra were taken at Perkin Elmer Spectrum One FT-IR spectrometer. Mass spectra were recorded on Ultima Fourier Transform and Varian 711 mass spectrometers. Elemental analyses were recorded on the Instrumental Analysis Laboratory of the TUBITAK Marmara Research Centre. Electronic spectra were taken at Scinco SD 1000 single-beam ultraviolet-visible (UV-vis) spectrophotometer at room temperature. 4-nitrophthalonitrile was synthesized according to a 
reported procedure (10). 4-(2-naphthoxy)phthalonitrile (1) was synthesized according to a procedure in the publications (11).

\section{Tetrakis(2-naphthoxy)phthalocyaninatopalladium(II) (2)}

4-(2-naphthoxy) phthalonitrile (1) $(0.270 \mathrm{~g}, 1 \mathrm{mmol})$ and $\mathrm{PdCl}_{2}(0.044 \mathrm{~g}, 0.25 \mathrm{mmol})$ in $2.5 \mathrm{~mL}$ of $\mathrm{N}, \mathrm{N}$-(dimethylamino)ethanol (DMAE) were mixed in a glass tube. The mixture was stirred and heated at $145^{\circ} \mathrm{C}$ for $24 \mathrm{~h}$ under $\mathrm{N}_{2}$. The resulting blue mixture was cooled to room temperature and the crude product was precipitated by addition of water. It was filtered off and the precipitate was successively washed with water and cold acetone, cold ethanol, and lastly with n-hexane, and then dried in vacuo. Finally, pure palladium(II) phthalocyanine compounds were purified by chromatography on silica gel using dichloromethane/ethanol (25:1) mixture as eluent. The yield was $122 \mathrm{mg}(41.21 \%)$. Melting point is over $200{ }^{\circ} \mathrm{C}$. Anal. calc. for $\mathrm{C}_{72} \mathrm{H}_{40} \mathrm{~N}_{8} \mathrm{O}_{4} \mathrm{Pd}: \mathrm{C}, 72.82 ; \mathrm{H}$, 3.39; N, 9.44. Found: C, 72.86; H, 3.36; N, 9.42\%; FT-IR, $\left(\mathrm{cm}^{-1}\right): 3055(\mathrm{Ar}-\mathrm{H}), 1459,1220$, 1155, 964; UV-Vis $\lambda_{\max }(\mathrm{nm})$ (log $\varepsilon$ ) in DMSO: 681 (4.81), 340 (4.71), 290 (4.97); MS: m/z $1187.012[\mathrm{M}]^{+}, 1205.977\left[\mathrm{M}+\mathrm{H}_{2} \mathrm{O}\right]^{+}$

\section{Preparation of PdPc (2) thin films}

Thin films were prepared in the following way: PdPc were dissolved in chloroform (CF), tetrahydrofuran (THF), dimethylformamide (DMF), and dimethyl sulfoxide (DMSO) by stirring with for $10 \mathrm{~min}$. The resulting solutions were blue and homogeneous. The same amount of PdPc was used for each solvent and the concentration of all solutions was $10 \mathrm{mg} / \mathrm{mL}$. Films were prepared on pre-cleaned microscope glass substrates by rotating them on a spin coater at a speed of $2000 \mathrm{rpm}$ for $15 \mathrm{~s}$. Finally, the films were dried at room temperature prior to optical measurement. Transmittance and absorbance spectra of the films were obtained by using a Thermo Scientific GENESYS 10S UV-vis spectrophotometer in the wavelength range of 200$1000 \mathrm{~nm}$.

\section{RESULTS AND DISCUSSION}

\section{Synthesis and characterization}

The starting compound of 4-(2-naphthoxy) phthalonitrile (1) was a base-catalyzed aromatic nitro displacement of 4-nitrophthalonitrile with $\beta$-naphthol (11). Conversion of 4-(2-naphthoxy) phthalonitrile (1) into related palladium(II) phthalocyanine (2) was achieved in $(\mathrm{N}, \mathrm{N}$ (dimethylamino)ethanol) as a high boiling solvent in the presence of metal salt $\left(\mathrm{PdCl}_{2}\right)(\mathrm{Scheme}$ 1) $(12,13)$. Tetrakis(2-naphthoxy)phthalocyaninatopalladium(II) (2) was characterized by FTIR, mass, elemental analysis and UV-Vis spectroscopic techniques. The palladium(II) phthalocyanine product in this study is a mixture of positional isomers. It is because the phthalonitrile precursor has a single substituent. The positional isomers were hardly separated by chromatography (14). 


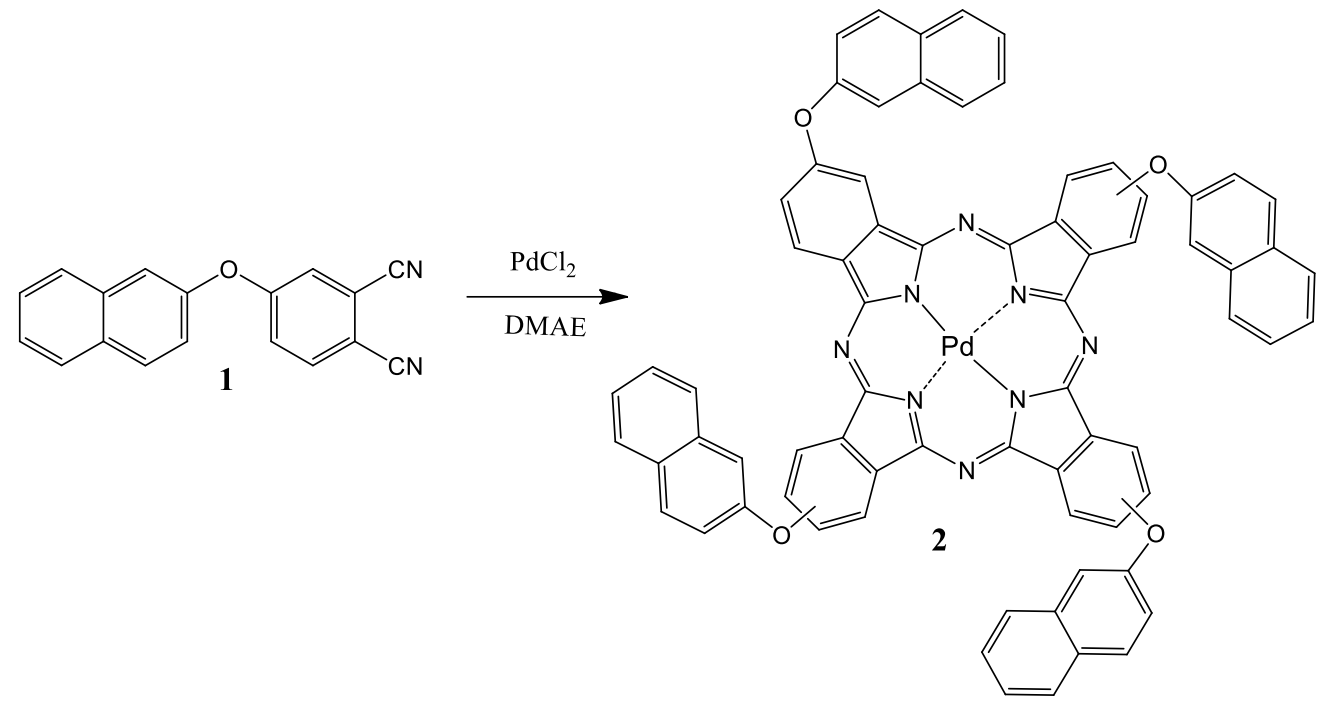

Scheme 1. Synthetic route of tetrakis(2-naphthoxy)phthalocyaninatopalladium(II) (2).

In the FT-IR spectrum of palladium(II) phthalocyanine (2), the typical C $\equiv N$ stretch at $\sim 2300$ $\mathrm{cm}^{-1}$ of 4-(2-naphthoxy) phthalonitrile (1) disappeared. Palladium(II) phthalocyanine (2) has expected IR vibration peaks due to aromatic $\mathrm{CH}$ stretching at $3050 \mathrm{~cm}^{-1}$. The FT-IR spectrum for palladium(II) phthalocyanine (2), showed transmittance peaks around 1459, 1220, 1155 and $964 \mathrm{~cm}^{-1}$ which may be assigned to phthalocyanine skeletal vibrations. The elemental analysis result of palladium(II) phthalocyanine (2) is consistent with the suggested structure. The mass spectrum of palladium(II) phthalocyanine (2) has the expected mass. The molecular ion peaks of 2 was observed at $\mathrm{m} / \mathrm{z}=1187.012[\mathrm{M}]^{+}$and $1205.977\left[\mathrm{M}+\mathrm{H}_{2} \mathrm{O}\right]^{+}$(Figure 1). The electronic absorption spectra of palladium(II) phthalocyanine (2), recorded in DMSO, show an intense $Q$ bands at $681 \mathrm{~nm}$ (Figure 2). The B bands of 2 come out in the UV region around $340 \mathrm{~nm}$. An intense broad electronic absorption band resulting from the $n-\Pi^{*}$ transition of naphthalene groups emerge in the UV region at about $290 \mathrm{~nm}$ for 2 (15).

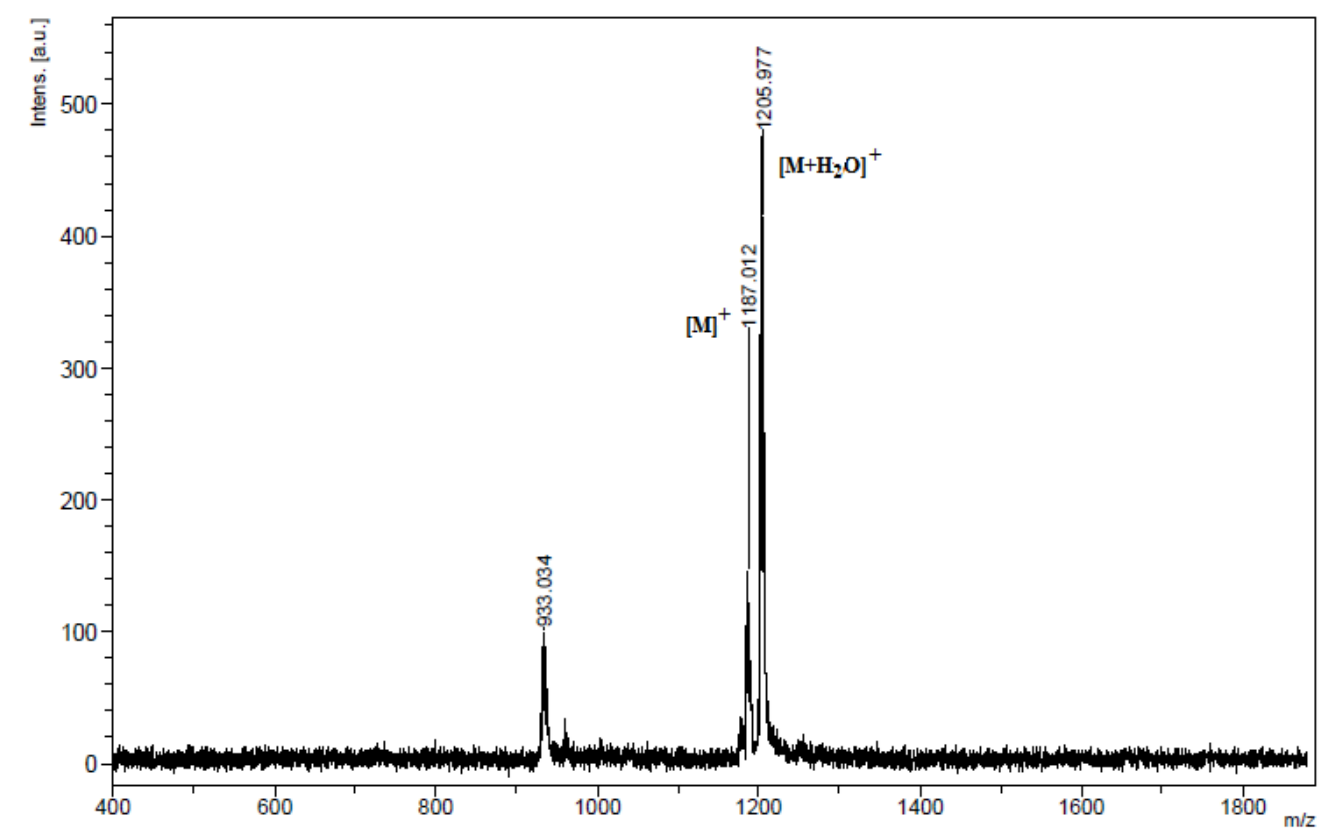

Figure 1: Mass spectrum of tetrakis(2-naphthoxy)phthalocyaninatopalladium(II) (2). 


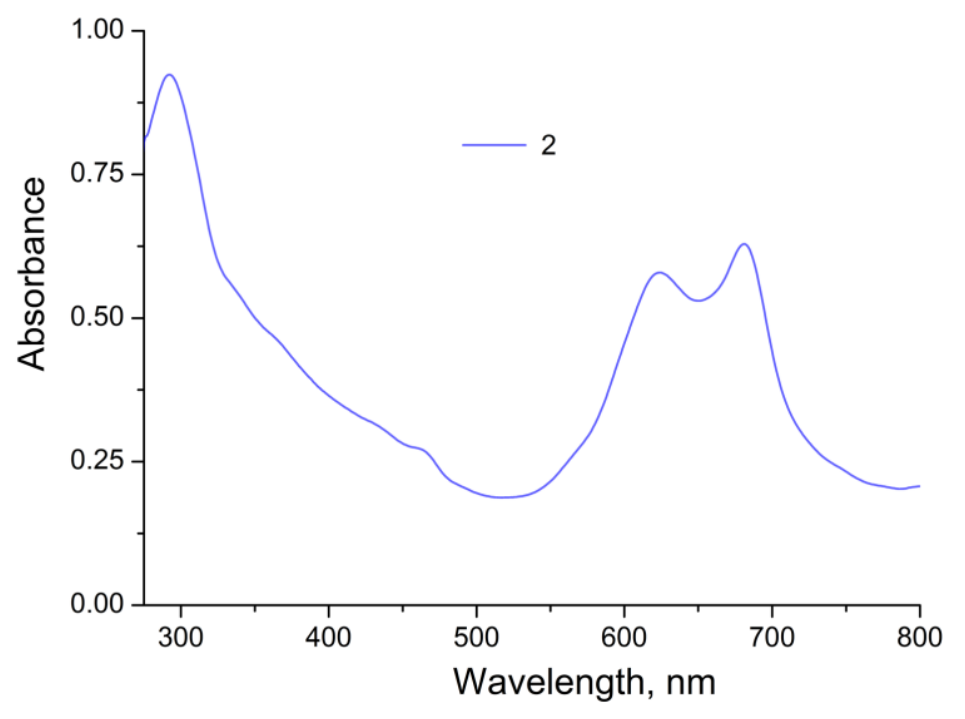

Figure 2: Absorption spectrum of (2) in DMSO $\left(1.0 \times 10^{-5} \mathrm{M}\right)$.

\section{Optical results}

The films prepared using DMF and DMSO solvents did not adhere completely to the surface but the film surfaces prepared using CF and THF solvents were found to be homogeneously coated. Transmittance spectra of the PdPc (2) films which were prepared by dissolving PdPc (2) in various solvents are shown in Figure 3. The peaks in the specific $Q$ band region of PdPc (2) were best seen in films prepared using CF and THF solvents. Nevertheless, no picture was found in this region of the films prepared using DMF and DMSO solvents. This may be attributed to their low adhesion to the surface, or having less solubility of them compared to other two solvents and there may not be enough PdPc (2) on the surface of the films prepared with these solvents. The transmittance value of all films in the visible region and near infrared region is above $80 \%$. It has been found that the solvents used to prepare PdPc (2) thin films have a significant effect on the transmittance and characteristic peaks in the $\mathrm{Q}$ band region of the spectrum. This is also clearly seen in Figure 4, in which the absorption spectrum is shown. There are studies showing that the amount of absorption in the $\mathrm{Q}$ band region can be adjusted by the concentration of the solvent and can be used for optical windowing purposes (9). In this study, it is seen that the use of different solvents is also effective in this region. 


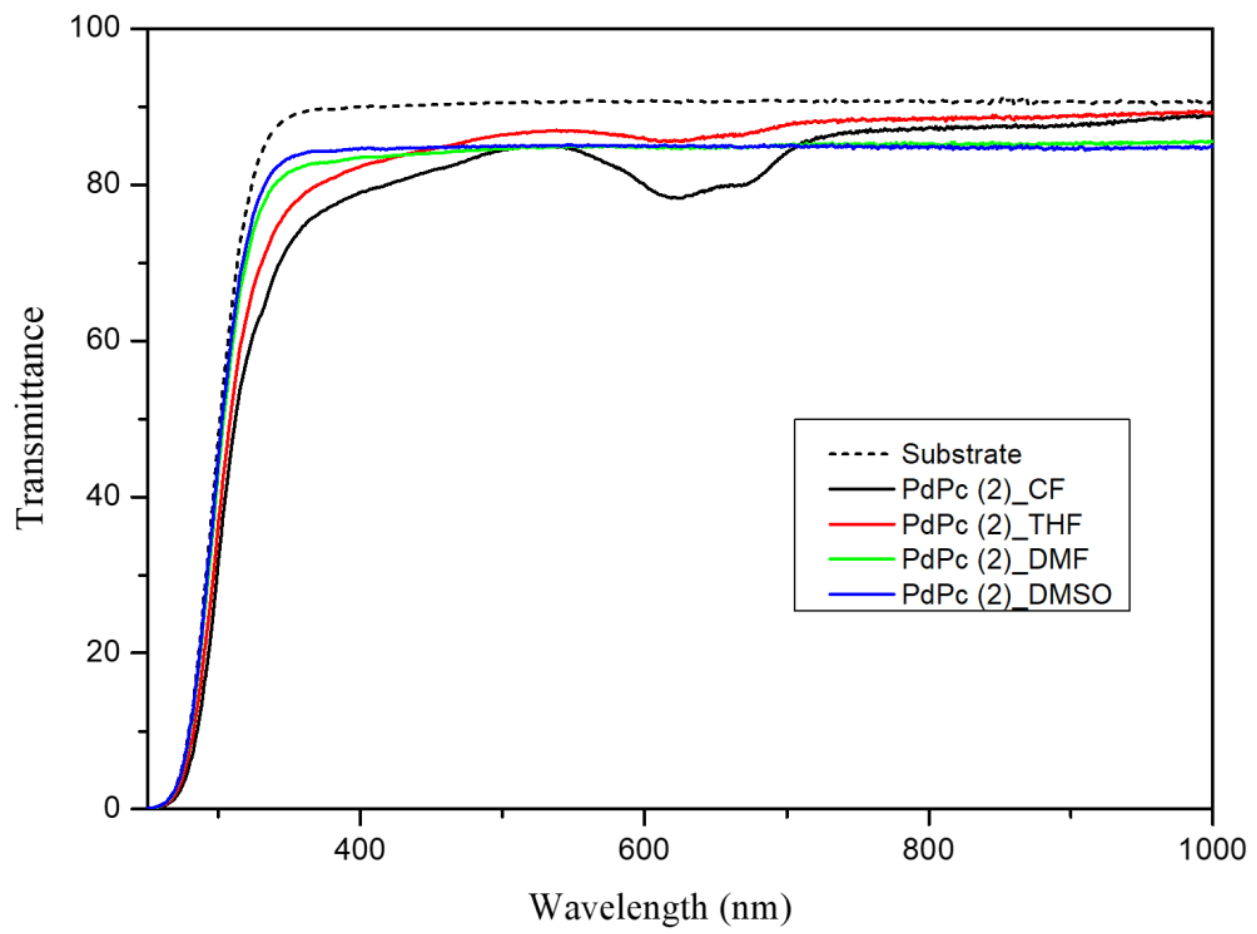

Figure 3: Transmittance spectra of PdPc (2) thin films as a function of solvents.

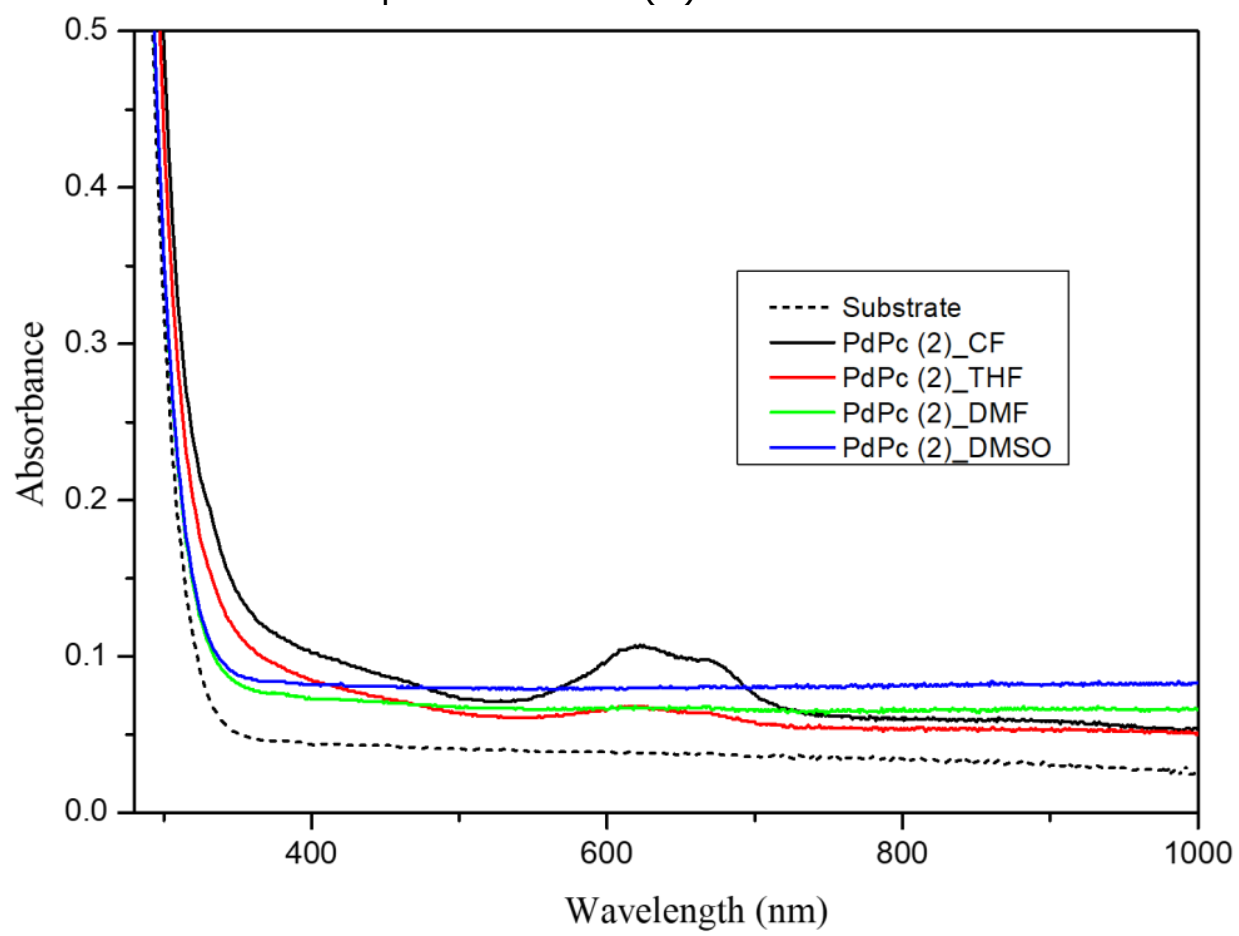

Figure 4: Absorption spectra of PdPc (2) thin films as a function of solvents.

Optical energy band gap of PdPc (2) films were calculated according to the following relation (16):

$$
\alpha=A\left(h v-E_{g}\right)^{m}
$$

where $A$ is a constant, and $m$ determines the type of the transition which is equal to $1 / 2$ for direct allowed transitions. Extrapolation of the linear part to photon energy axis (Figure 5) gives 
the energy band gaps of the films. They were found about 4.48, 4.51, 4.55 and $4.55 \mathrm{eV}$ for PdPc (2) films as a function of solvents CF, THF, DMF and DMSO, respectively. It has been observed that films prepared using different solvents have changed the value of the energy band gaps.

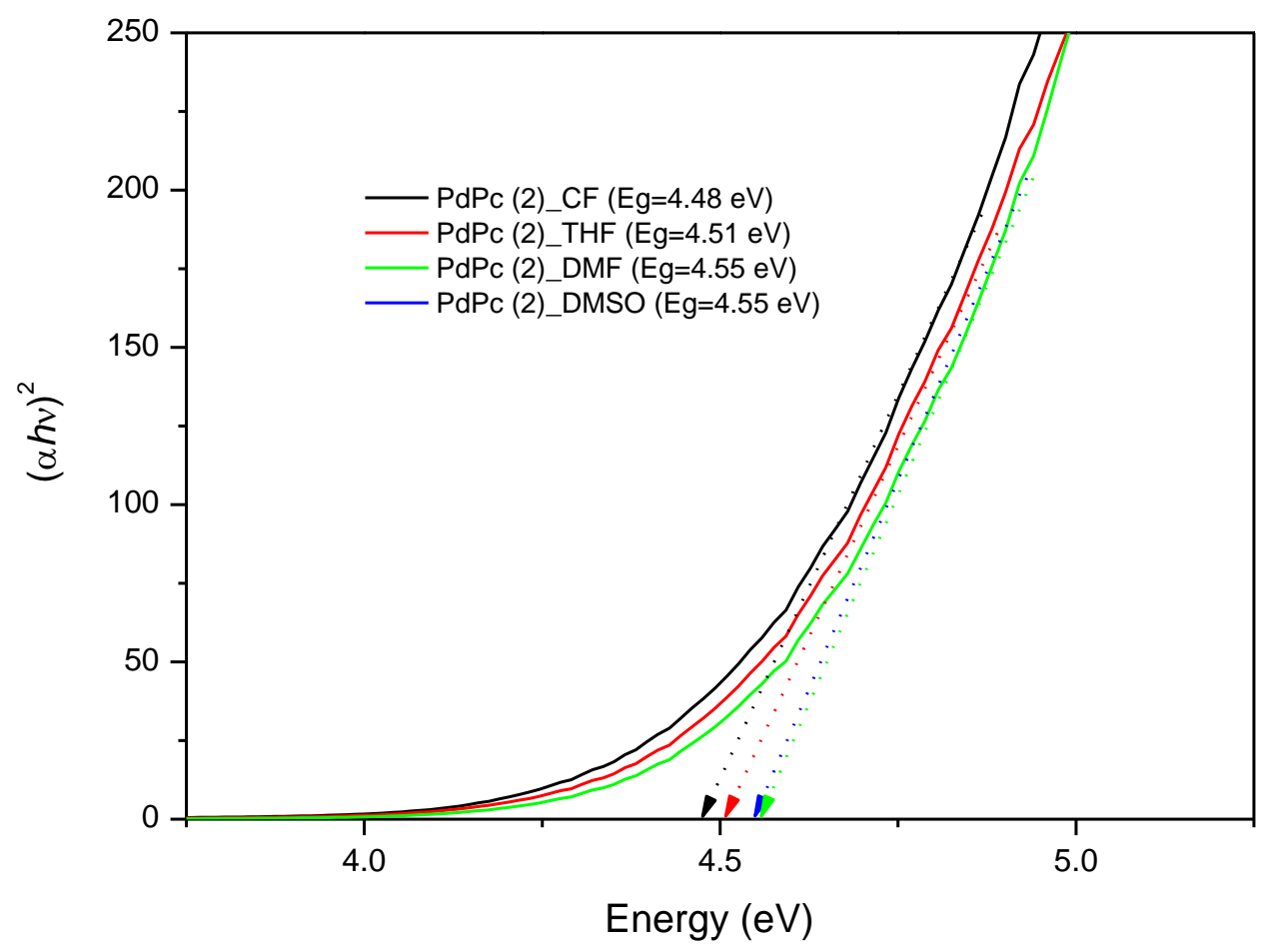

Figure 5: Relation between vs. photon energy for PdPc (2) thin films as a function of solvents.

\section{CONCLUSION}

In summary, we have synthesized and characterized peripherally $\beta$-naphthoxy substituted palladium(II) phthalocyanine (2). Thin films of palladium(II) phthalocyanine were prepared using different organic solvents by spin coating technique. The transmittance value of all films in the visible region and near infrared region is above $80 \%$. The peaks in the specific $\mathrm{Q}$ region of PdPc (2) were best seen in films prepared using CF and THF solvents. It has been observed that energy band gaps of the films may be modulated by using different solvents. PdPc (2) thin films prepared by using different organic solvents especially CF exhibit a good absorption in the visible region of electromagnetic spectrum hence they could be good candidate for various device applications such as optical filters.

\section{ACKNOWLEDGEMENT}

This work was supported by the Research Fund of the Istanbul Technical University. 


\section{REFERENCES}

1. McKeown NB. Phthalocyanine Materials: synthesis, structure, and function. Cambridge University Press: Cambridge, 1998.

2. Leznoff CC, Lever ABP. Phthalocyanines: properties and applications. Vols. 1-4. Eds. VCH Publishers: New York, USA, 1989.

3. Özçeşmeci İ, Güner S, Okur Aİ, Gül A. New phthalocyanines containing bulky electron rich substituents. J. Porphyrins Phthalocyanines. 2007; 11(07): 531-6.

4. Kurt Ö, Özçesmeci İ, Gül A, Koçak MB. Synthesis and photophysical properties of novel hexadecasubstituted phthalocyanines bearing three different groups. J. Organomet. Chem. 2014; 754: 8-15.

5. Seshan K. Handbook of Thin-Film Deposition Processes And Techniques, W. Andrew Publishing: Norwich, New York, U.S.A., 2002.

6. Özçesmeci İ, Sorar İ, Gül A. Optical studies on phthalocyanines substituted with phenylazonaphthoxy groups. Philos. Mag. 2016; 96(28): 2986-99.

7. Özçeşmeci M, Sorar İ, Hamuryudan E. Synthesis, optical and structural studies of tetrakis [4-( $2^{\prime}, 3^{\prime}, 4^{\prime}$, 5', 6'-pentafluorobenzyloxy) benzyloxy]-substituted metallo-phthalocyanines. Synth. Met. 2012; 162: $154-61$.

8. Sorar İ, Şener MK, Tepehan F, Gül A. Structural and optical studies of tetra (tricarbethoxy)-substituted metallophthalocyanines. Thin Solid Films. 2008; 516: 2894-98.

9. Özçesmeci İ, Sorar İ, Gül A. Zinc (II) phthalocyanine as an optical window for visible region. Inorg. Chem. Commun. 2011; 14: 1254-57.

10. Young JG, Onyebuagu WJ. Synthesis and Characterization of Di-disubstituted Phthalocyanines, Org. Chem. 1990; 55: 2155-59.

11. Yenilmez HY, Özçeşmeci İ, Okur Aİ, Gül A. Synthesis and characterization of metal-free and metallophthalocyanines with four pendant naphthoxy-substituents. Polyhedron. 2004; 23: 787-91.

12. Özçeşmeci M. Synthesis, photophysical and photochemical properties of metal-free and zinc (II) phthalocyanines bearing a-naphtholbenzein units. J. Organomet. Chem. 2014; 767: 16-21.

13. Özçeşmeci İ, Yerli Y, Okur Aİ, Gül A. Synthesis and EPR studies of a near infrared absorbing tetrakis(2naphthoxy)vanadyl-phthalocyanine. Inorg. Chem. Commun. 2009; 12: 625-27.

14. Hanack M, Schmid G, Sommeraner M, Chromatographic separation of the four possible structural isomers of a tetrasubstituted phthalocyanine: tetrakis(2-ethylhexyloxy)phthalocyaninatonickel(II). Angew. Chem. Int. Ed. Engl. 1993; 32 (10): 1422-24.

15. Özçesmeci İ, Koca A, Gül A. Synthesis and electrochemical and in situ spectroelectrochemical characterization of manganese, vanadyl, and cobalt phthalocyanines with 2-naphthoxy substituents. Electrochim. Acta. 2011; 56: 5102-14.

16. Tauc J, Grigorovici R, Vancu A, Optical properties and electronic structure of amorphous germanium. Phys. Status Solidi. 1966; 15: 627-37. 